\title{
DMD-based rapid 3D bioprinting for precision tissue engineering and regenerative medicine
}

Claire Yu, Shangting You, Wei Zhu, Bingjie Sun, Shaochen Chen

Claire Yu, Shangting You, Wei Zhu, Bingjie Sun, Shaochen Chen, "DMDbased rapid 3D bioprinting for precision tissue engineering and regenerative medicine," Proc. SPIE 11294, Emerging Digital Micromirror Device Based Systems and Applications XII, 1129405 (28 February 2020); doi: $10.1117 / 12.2550340$

SPIE. Event: SPIE OPTO, 2020, San Francisco, California, United States 


\title{
DMD-based Rapid 3D Bioprinting for Precision Tissue Engineering and Regenerative Medicine \\ Claire Yua $u^{\mathrm{a}}$, Shangting You ${ }^{\mathrm{a}}$, Wei Zhua, Bingjie Sun ${ }^{\mathrm{a}}$, Shaochen Chen*a \\ a Department of NanoEngineering, University of California, San Diego, 9500 Gilman Drive, La Jolla, California, 92093, USA.
}

\begin{abstract}
DMD-based 3D printing is a powerful tool for making high-resolution biomimetic functional tissues and organs with various biomaterials for tissue engineering and regenerative medicine. A plethora of tissues have been fabricated using this technology including liver, heart, lung, kidney, blood vessels, cartilage, and placenta. In this article, we show prevascularization of the artificial tissue constructs using DMD-based 3D printing, which is essential to maintain the long term viability and function of a thick tissue. We also show a 3D printed biomimetic hepatic model that recapitulates the microarchitecture as well as the heterogeneous cell population of various cell types in the native liver tissue. It is important for the biomaterials to mimic the native microenvironment. Finally, we demonstrate that $3 \mathrm{D}$ printed tissue-specific decellularized extracellular matrix can improve cell response and behavior.
\end{abstract}

Keywords: 3D printing, tissue engineering, biomaterials, DMD

\section{INTRODUCTION}

With the emergence of new technologies in the field of tissue engineering and regenerative medicine, the prospect of making personalized functional human tissues and organ substitutes can be realized. Among the different strategies and techniques available to tissue engineers, the area of 3D bioprinting has created a paradigm shift by giving researchers a versatile tool to build a wide range of complex tissues that recapitulate the in vivo cellular heterogeneity, microarchitectures, biophysical properties, and biochemical constituents. More specifically, 3D bioprinting provides a method to precisely control the patterning of different cell types and biomaterials such that they represent the biophysical arrangement of native tissues. To date, a plethora of tissues have been fabricated using 3D bioprinting technology including but not limited to the liver, heart, lung, kidney, blood vessels, cartilage, and placenta ${ }^{1-7}$. In turn, these bioprinted tissues have important implications in various biological and medical disciplines ranging from physiologically relevant models for elucidating biological mechanisms and drug screening, to integration with biosensors and microfluidics for lab-on-achip devices as well as transplantable tissues for clinical applications. In this article, we will discuss the current state of 3D printing systems followed by highlighting our current work on 3D bioprinted prevascularized tissue constructs as well a 3D bioprinted hepatic model, and finally discussing the future of next-generation decellularized extracellular matrix $(\mathrm{dECM})$ bioinks as tissue-specific biomaterials to facilitate the development of more physiologically-relevant tissues.

\section{DMD-BASED 3D BIOPRINTING}

Currently, there are several different 3D printing platforms available which can be categorized as inkjet-based printers, extrusion-based printers, laser-based stereolithography, and digital micro-mirror array device (DMD)-based printers with each varying in terms of print resolution, speed, and biomaterial compatibility. One of the most commonly used is the traditional 3D inkjet printer that was first adopted from the common desktop ink-based paper printers, in which biomaterials and cells are deposited in a raster-like manner ${ }^{8}$. By using acoustic, thermal, or electrostatic forces, inkjet printers deposit materials drop-by-drop through a fine nozzle onto a receiving substrate placed on a movable stage. These printers are relatively low cost, capable of micron scale printing resolutions $(\sim 50 \mu \mathrm{m})$, and limited to using low viscosity materials ${ }^{9}$. Similarly, extrusion-based printers work by depositing materials through a nozzle tip using pneumatic or mechanical pressure upon which the material solidifies on contact with the receiving substrate. These printers can achieve feature size from $5 \mu \mathrm{m}$ to a few millimeters wide with moderate speeds of fabrication, and can accommodate a larger range

Emerging Digital Micromirror Device Based Systems and Applications XII, edited by John Ehmke, Benjamin L. Lee,

Proc. of SPIE Vol. 11294, 1129405 - @ 2020 SPIE · CCC code: 0277-786X/20/\$21 - doi: 10.1117/12.2550340 
of material viscosities and higher cell densities compared to inkjet printers ${ }^{10,11}$. Furthermore, laser-based stereolithography utilizes laser-induced photopolymerization to polymerize materials in a contactless iterative fashion. Here, microstructures with overhanging features can be produced possessing micro and nanoscale resolutions ${ }^{12,13}$. While these printing systems are well-established, they have shortcomings in terms of fabrication speed, limited range of compatible materials, potential for clogging or decreased cell viability due to shear stress, and scalability.

In more recent years, DMD-based 3D bioprinters have enabled a more rapid approach for the microfabrication of complex structures as well as improved spatial resolution not attainable by the nozzle and inkjet based printing systems. In general, slices of the object are digitized into a series of light patterns which are then projected by a DMD to a prepolymer solution. The DMD containing millions of micro-mirrors (e.g. $1920 \times 1080)$ for light projection enables massively parallel printing without the need of point-by-point scanning. By using this technique an entire plane of an object can be fabricated at once, and as the z-stage moves continuously, the 3D structure can be built continuously, resulting in smooth features and the elimination of artifacts between adjacent layers normally encountered with traditional 3D printing approaches. For instance, both dynamic optical projection stereolithography (DOPsL)-based and continuous liquid interphase production (CLIP)-based printing technologies adopt these principles. High resolutions of 3-5 $\mu \mathrm{m}$ in the $\mathrm{x}-\mathrm{y}$ direction can be achieved using the DOPsL-based method ${ }^{14}$. Furthermore, CLIP-based printers have been shown to be able to form complex overhanging structures with less than $100 \mu \mathrm{m}$ resolution and produce large constructs on the centimeter scale ${ }^{15}$. In general, DMD-based printers can produce printed structures with micron scale resolution in mere seconds and have been demonstrated to be well-suited for cell encapsulation to form biomimetic tissue constructs ${ }^{1,5,12,14,15}$.

\section{3D BIOPRINTING OF PREVASCULARIZED TISSUE CONSTRUCTS}

As the 3D bioprinting field advances towards the generation of larger and more complex tissues for in vivo transplantation, there is a critical need for the ability to vascularize these tissue constructs in order to maintain long term viability and function ${ }^{16}$. One approach is incorporate pro-angiogenic growth factors to induce host vascularization into the tissue construct, however, this process is slow and leads to decreased cell viability after initial transplantation ${ }^{17,18}$. As such, it has been recognized that a preexisting vascular network would be a more favorable strategy to accelerate the vascularization process by allowing the host to directly anastomose to the graft and improve cell viability, blood perfusion, and vascularization ${ }^{16,19}$. While many methods have been demonstrated to create prevascularized tissues such as with the use of sacrificial materials and micromolding techniques, they are time consuming and require multiple steps which may not lend well for large-scale production. In our work, we utilized a rapid DMD-based microscale continuous optical bioprinter $(\mu \mathrm{COB})$ to produce prevascularized tissue constructs directly in a one-step manner by patterning endothelial cells into complex hierarchal vascular branched networks (Fig. 1) ${ }^{5}$. Specifically, we bioprinted a vascular bioink composed of human umbilical endothelial cells (HUVECs) and 10T1/2 cells (50:1 ratio) in a naturally-derived prepolymer solution comprised of photopolymerizable gelatin methacrylate (GelMA) and glycidal methacrylate hyaluronic acid (GMHA) with lithium phenyl-2,4,6 trimethylbenzoylphosphinate (LAP) as the photoinitator. The entire printing process can be accomplished in less than one minute using our technique with high resolution and complex microarchitecture of heterogeneous cellular components (Fig. 1 D-I), such that endothelial regions were patterned within the surrounding hepatocellular regions containing HepG2 cells.

After one-week in vitro culture, the bioprinted prevascularized tissue constructs maintained good structural integrity along with high cellular viability of over $85 \%$. Furthermore, immunohistochemical stains for human-specific CD31 and $\alpha$-smooth muscle actin (Fig. $1 \mathrm{~J}$-L) of the hierarchal bioprinted tissue constructs with varying vessel diameters ranging from approximately 50 to $250 \mu \mathrm{m}$, demonstrated the induction of $10 \mathrm{~T} 1 / 2$ cells adopting a pericyte phenotype with the HUVECs lining the outer walls of the vessel patterns and forming a lumen-like structure in one week. 

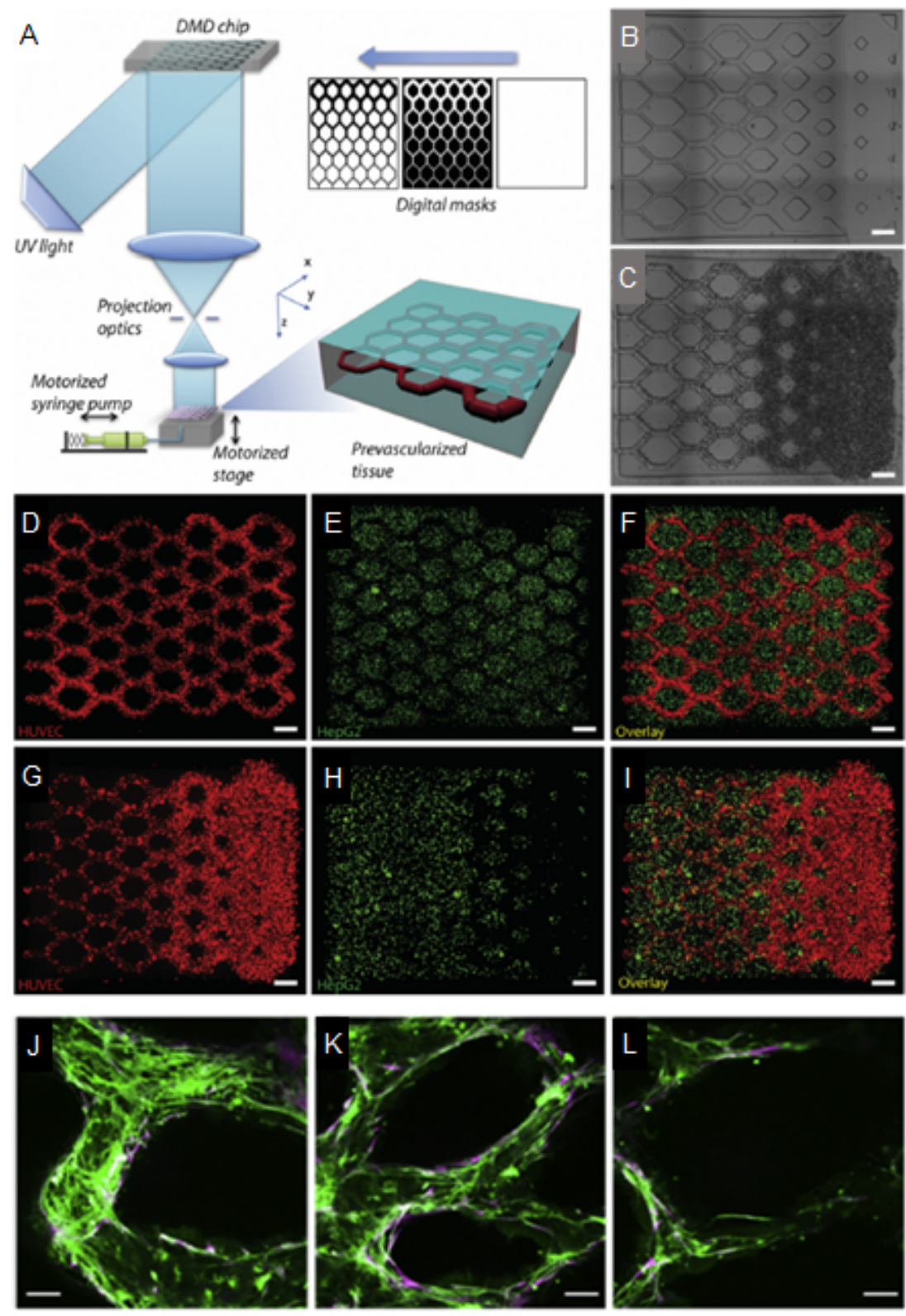

Figure 1: A) Schematic for the 3D bioprinting of prevascularized tissues and corresponding bright field images of printed (B) acellular and (C) cellularized constructs encapsulated with HUVECs and 10T1/2 (50:1 ratio). (D-F) Fluorescent images of prevascularized tissue constructs with uniform channel diameters of (D) HUVECs (red) in the vascular regions and HepG2 cells (green) in the surrounding regions. (G-I) Fluorescent images of prevascularized tissue constructs with gradient channel diameters. Scale bars $=250 \mu \mathrm{m}$. (J-L) Representative confocal images of endothelial networks formed after oneweek in vitro culture with CD31 positive HUVECs (green) and $\alpha$-smooth muscle actin ( $\alpha$-SMA) positive pericyte-like $10 \mathrm{~T} 1 / 2 \mathrm{~s}$ (purple) patterned in vessels of different diameters. Scale bars $=100 \mu \mathrm{m}$. (Copyright 2017 Elsevier.) 

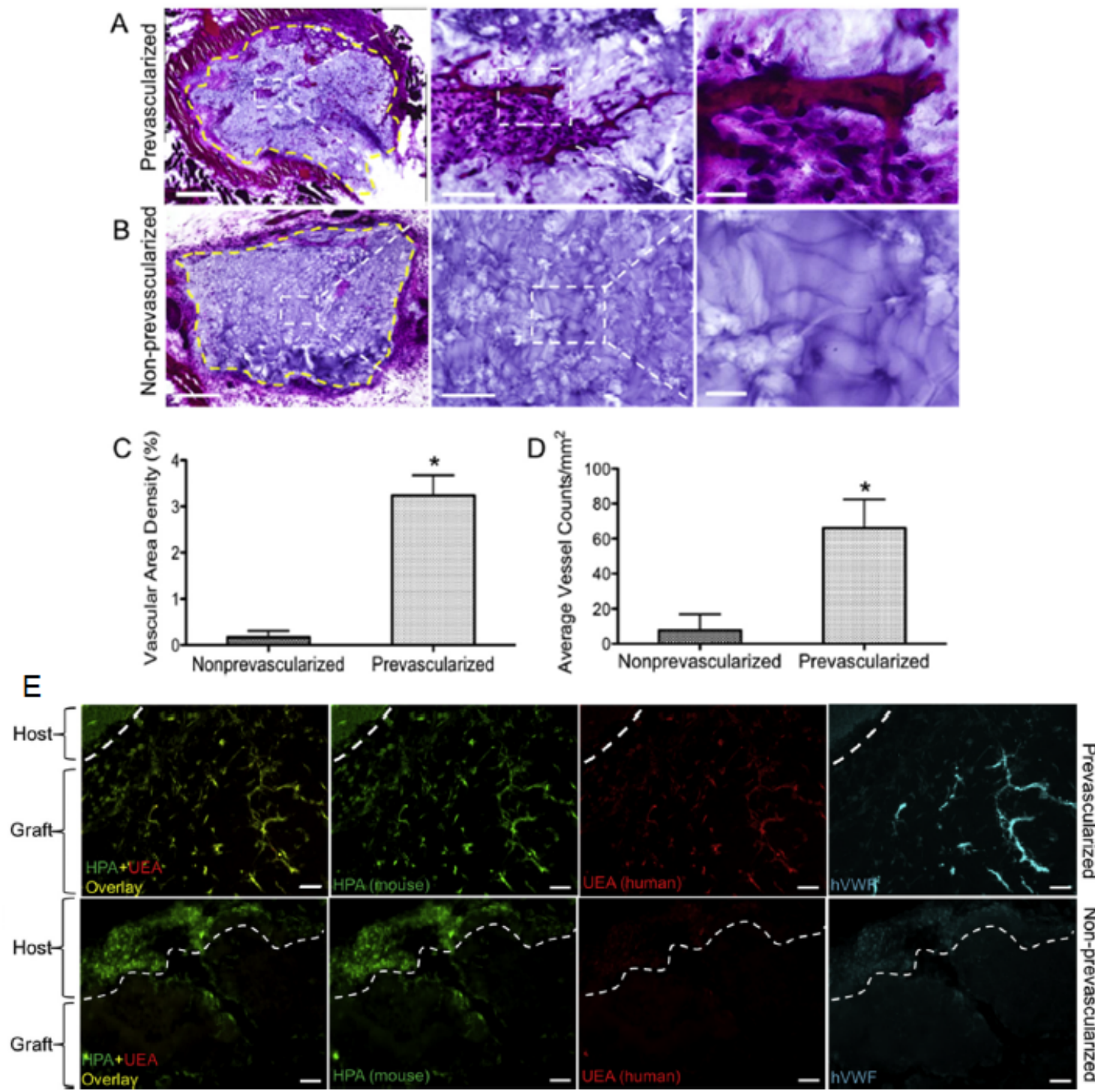

Figure 2: Representative H\&E stained images of implanted (A) prevascularized tissue constructs showing significant vessel formation and blood within the lumens, and (B) non-prevascularized tissue constructs showing limited presence of blood vessels. Scale bars =(A) $500 \mu \mathrm{m}$ (left), $100 \mu \mathrm{m}$ (middle), $25 \mu \mathrm{m}$ (right); (B) $500 \mu \mathrm{m}$ (left), $100 \mu \mathrm{m}$ (middle), $25 \mu \mathrm{m}$ (right). (C) Quantification of percentage vascular area density and (D) average vessel counts per $\mathrm{mm}^{2}$ of grafted tissue construct after 2-week subcutaneous implantation. Error bars represent SEM for all data points and * = significance between the prevascularized and non-prevascularized groups, $p<0.05(n=6)$. (E) Representative fluorescent images of showing chimeric presence of mouse-specific lectin (HPA) and human-specific lectin (UEA) in the implant region after two weeks postsurgery for the prevascularized tissue constructs. Further staining of hVWF confirms the endothelial network formed by human origin HUVECs. In the non-prevascularized tissue, HPA stains the host tissue and minor regions of the graft area, no UEA or hVWF staining is observed. Scale bars $=100 \mu \mathrm{m}$. (Copyright 2017 Elsevier.)

To assess the efficacy of our bioprinted prevascularized tissue constructs to form endothelial networks in vivo, we implanted them subcutaneously in the dorsal region of severe combined immunodeficiency (SCID) mice. After two weeks post-surgery, histological images revealed an increased number of visibly mature endothelial vasculature with red blood cells present within the lumens (Fig. 2A) as compared to non-prevascularized tissue controls (Fig. 2B). This is further supported by measurements of the vascular density and average vessels counts per area in the graft region showing a significant increase for the prevascularized tissue constructs in both cases (Fig. 2C,D). To evaluate the anastomosis of the bioprinted prevascularized construct to the host vasculature, SCID mice were administered with mouse-specific lectin (HPA) and human-specific lectin (UEA) to differentiate between mouse and human endothelial cells, respectively. 
Fluorescent images shown in Fig. 2E indicated that regions within the prevascularized tissue implant stained positive for both HPA and UEA, thus confirming the presence of cells originating from the host and graft in the vessels. Further staining of human von Willebrand factor (hVWF) confirmed the endothelial network formed by human origin HUVECs. In contrast, for non-prevascularized tissue controls only HPA positive staining was observed in the host tissue regions and in the outer borders of the construct, and no UEA or hVWF staining was observed. Overall, these results demonstrate that the prevascularized networks integrated and anastomosed to the host vascular system and was able to support blood perfusion.

This study showcases a simple and efficient method of applying DMD-based 3D bioprinting techniques to rapidly fabricate intricate vascular networks directly within tissues constructs. The flexibility of our 3D bioprinting platform also allows the potential to engineer more complex heterogeneous tissues through the incorporation of other cell types and photopolymerizable biomaterials, in addition to the scalability of this approach to fabricate large tissue constructs. The biocompatibility and anastomosis to the host observed from the in vivo studies also demonstrates the significant potential of these prevascularized tissue constructs to serve as tissue grafts for the future goal of producing transplantable tissue or organ substitutes in tissue engineering and regenerative medicine.

\section{3D BIOPRINTING OF A BIOMIMETIC HUMAN IPSC-DERIVED HEPATIC MODEL}

One of the key aspects towards the creation of truly biomimetic tissues is the need to recapitulate the heterogeneous cell population composed of parenchymal cells, stromal cells, and other supportive nonparenchymal cells. This concept derives from the fact that multiple cell types co-exist in vivo and communicate through paracrine signaling with each other as well as their interactions with the surrounding extracellular matrix (ECM) microenvironment to form a functional tissue unit ${ }^{20}$. As such, 3D bioprinting technologies offer a precise way to pattern various cell types to essentially build a tissue using a bottom-up approach. While cell patterning is crucial, their sourcing is also of great importance in the context of creating physiologically relevant tissue models. The majority of printed tissues to date have been produced using established cell lines or animal-derived cell sources to serve as a proof-of-concept, however, these cells do not fully reflect the behavior and function of human primary cells ${ }^{21}$. With the advent of human induced pluripotent stem cells (hiPSCs) and their ability to differentiate into a multiple cell types including cardiac, liver, kidney, and neuronal cells, their use in 3D bioprinting would be of great importance due to their abundance and stability ${ }^{22}$.

To address these challenges as well as expand our work on prevascularized tissue constructs, our group 3D bioprinted the first in vitro biomimetic hepatic model that recapitulates the native microenvironment through the use of multiple human-derived cell types patterned in a complex microarchitecture. Our 3D hepatic triculture model incorporated hiPSC-derived hepatic progenitor cells (hiPSC-HPCs) in the lobular regions in conjunction with HUVECs and human adipose-derived stem cells (ADSCs) in the surrounding vascular network. Furthermore, two naturally-derived photopolymerizable hydrogels were chosen in which GelMA was used for the hepatic component and GelMA + GM-HA was used for the vascular component. After patterning the hiPSC-HPCs and the endothelial cells via a two-step printing process (Fig. 3A,B), the microscale hexagonal microarchitecture can be clearly observed to reflect the tissue-scale anatomical features and dimensions of the liver in our design (Fig. 3C). 


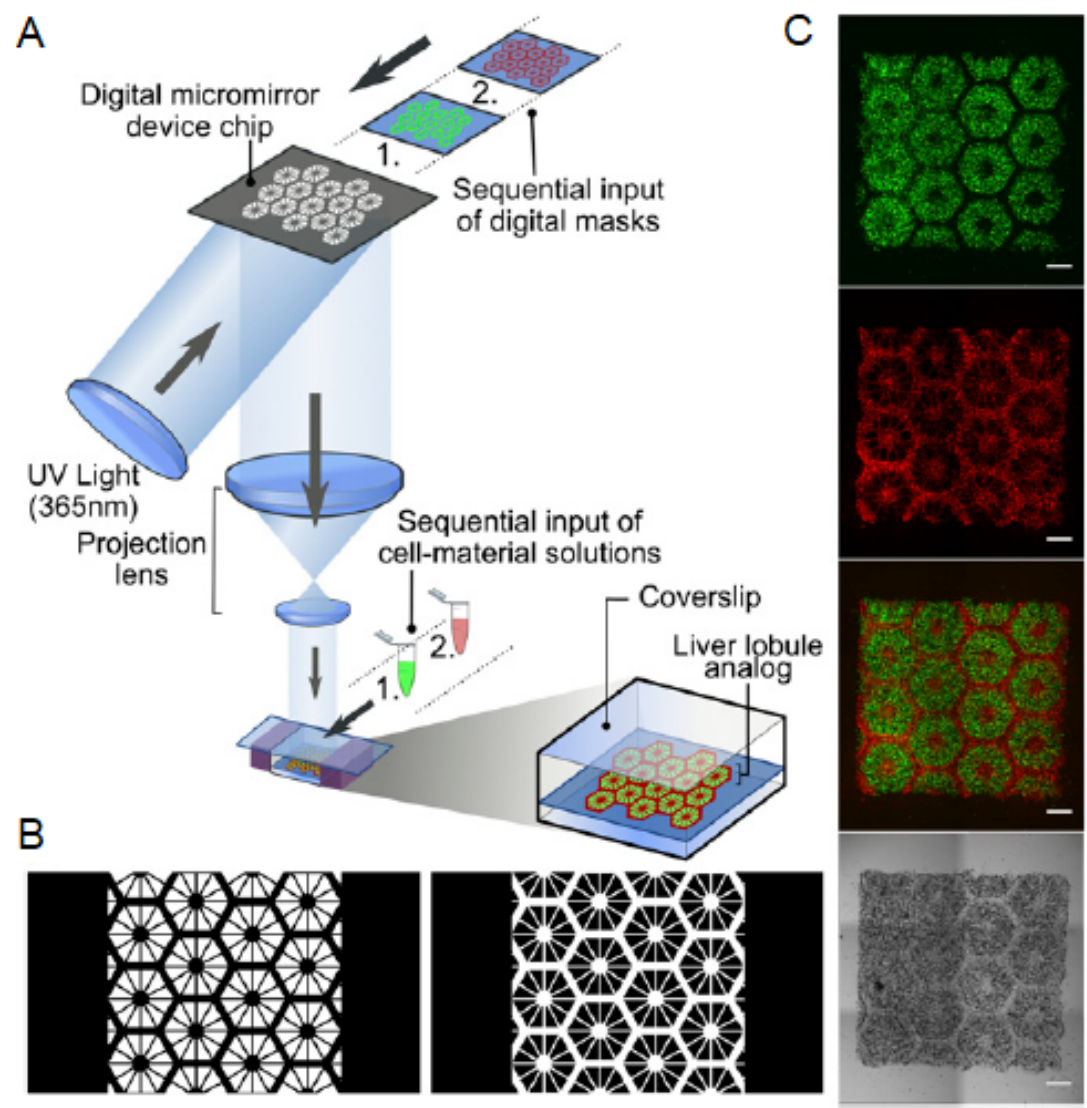

Figure 3: (A) Schematic of the two-step 3D bioprinting process to construct the triculture hepatic model. (B) Digital patterns of the hepatic lobule structure (left) and vascular regions (right) where polymerized areas are indicated in white. (C) Representative fluorescent images of the bioprinted hepatic model showing iPSC-HPCs (green) and the surrounding vasculature (red) immediately after fabrication. Scale bars $=500 \mu \mathrm{m}$. (Copyright 2016 National Academy of Sciences.)

To assess the structural integrity of the bioprinted 3D triculture hepatic construct, cells were fluorescently tracked over 10 days in vitro culture. Interestingly, the cells remained within each of their patterned regions for at least 10 days post-printing and integrated into a single construct over time (Fig. 4A). Moreover, immunohistochemical staining of albumin (ALB) and E-cadherin (E-cad) in the 3D hepatic triculture constructs revealed larger aggregation and spheroid formation over time as compared to hiPSC-HPC only controls (Fig. 4B). These observations suggested a higher degree of cell junction formation as well as maturation and functionality, which was further supported by the gene expression profile (Fig. 5A) demonstrating higher levels of the key liver markers: albumin (ALB), hepatocyte nuclear factor 4 alpha (HNFa), transthyretin (TTR), and alpha fetal protein (AFP) relative to the 3D hiPSC-HPC only and 2D monolayer controls. To further assess the functionality of our 3D hepatic triculture model, quantification of albumin concentration and urea production also confirmed that significantly higher secretion levels for both were observed across 20 days of in vitro culture compared to 3D hiPSC-HPC only and 2D monolayer controls (Fig. 5B,C). 

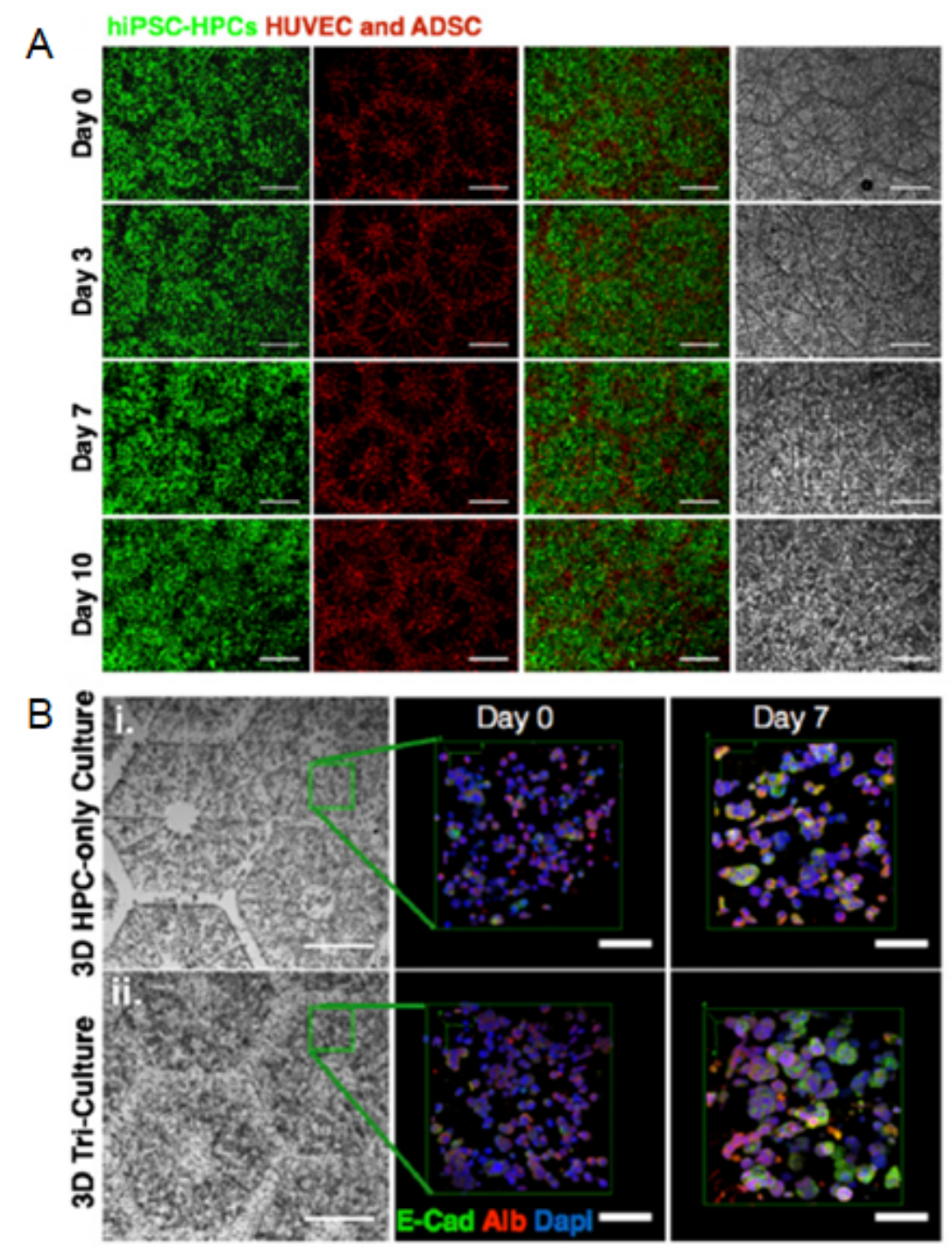

Figure 4: (A) Representative fluorescent images of the hepatic tissue constructs over 10 days in vitro culture showing preservation of the individual patterns for the hepatic and vascular regions over time. Scale bars $=500 \mu \mathrm{m}$. (B) Immunohistochemical stained images for E-cadherin (E-cad) and albumin (Alb) in the 3D iPSC-HPC only versus 3D hepatic triculture model. Scale bars $=500 \mu \mathrm{m}$ (bright field) and $100 \mu \mathrm{m}$ (fluorescent). (Copyright 2016 National Academy of Sciences.)

To evaluate the potential for our 3D hepatic triculture model to serve as platform for drug screening, the anabolic and catabolic functions of our model was assessed by examining the upregulation of expression of key CYP enzymes that are involved in liver drug metabolism (Fig. D) ${ }^{23}$. Baseline CYP levels without drug treatment showed that our 3D triculture model had significantly elevated levels of CYP3A4 expression and higher levels of CYP1A2, CYP2B6, CYP2C9, and CYP2C19 compared to the 3D hiPSC-HPC only and 2D culture controls. Upon introducing rifampicin, a known bactericidal antibiotic drug to induce hepatotoxicity ${ }^{24}$, our 3D triculture hepatic model exhibited increased CYP3A4, CYP2C9, and CYP2C19 expression in comparison to untreated controls. In contrast, no significant increases in the CYP enzyme expression was observed for the 3D hiPSC-HPC only and 2D culture controls. 


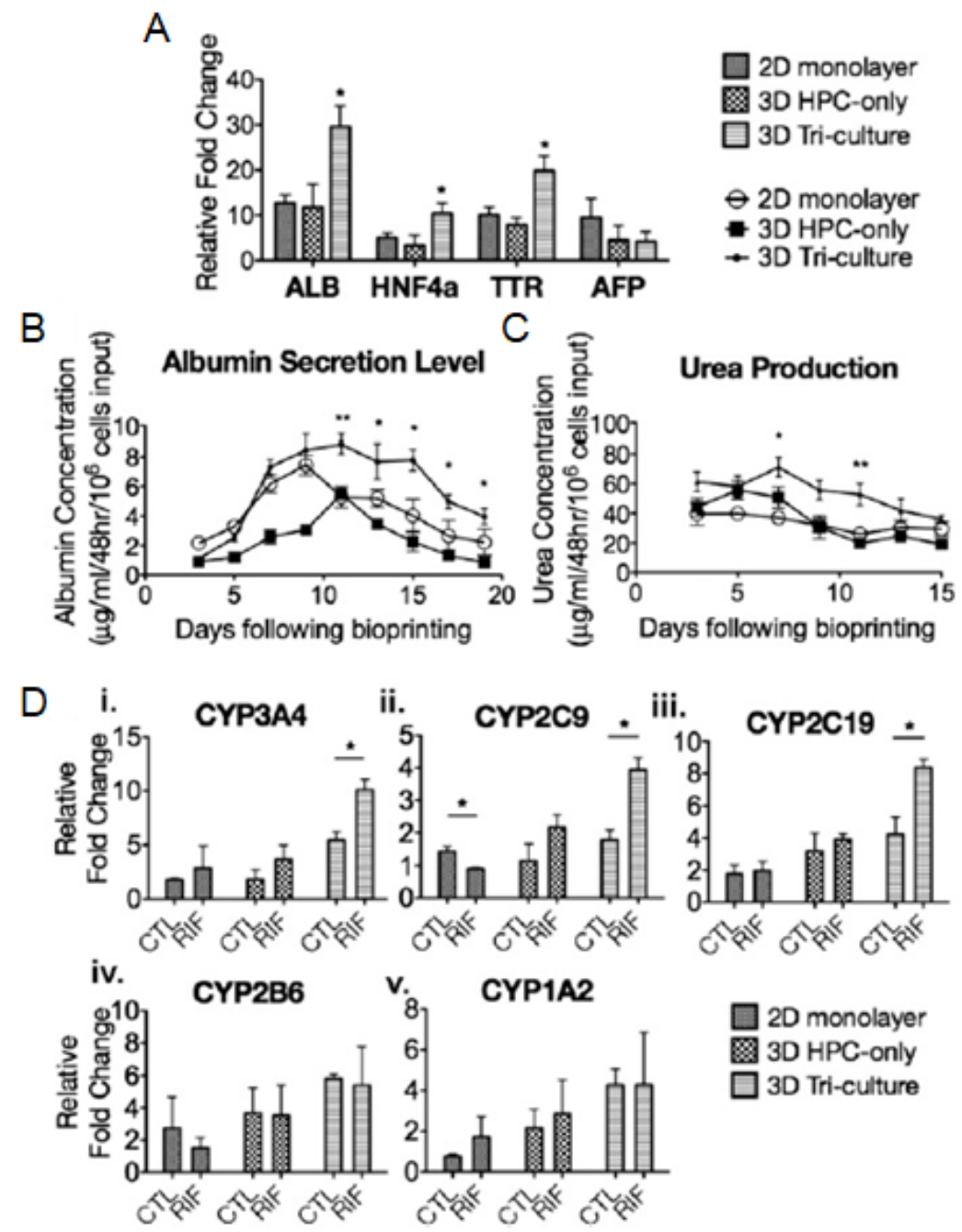

Figure 5: (A) Gene expression profile for the functional characterization of the 3D triculture hepatic model compared to the 3D HPC-only and 2D monolayer controls after one-week in vitro culture for the key markers: albumin (Alb), hepatocyte nuclear factor 4 alpha (HNFa), transthyretin (TTR), and alpha fetal protein (AFP). (B) Quantification of albumin secretion levels and (C) urea production over 15 days in vitro culture for each of the three groups. (D) Gene expression profile of (i) CYP3A4, (ii) CYP2C9, (iii) CYP2C19, (iv) CYP2B6, and (v) CYP1A2 for each group with and without (CTL) rifampicin (RIF) treatment after one-week in vitro culture. All groups were normalized to hiPSC-HPCs on day 12 of differentiation prior to 3D bioprinting. Error bars represent SEM for all data points with significance at $\mathrm{p}<0.05$ ( $\mathrm{n}=3$ ). (Copyright 2016 National Academy of Sciences.)

Overall, we demonstrated the utility of our DLP-based 3D bioprinter to produce a complex functional 3D biomimetic hepatic triculture model that recapitulates both the native liver microarchitecture and cellular diversity. More importantly, the improved albumin and urea secretion as well as drug response in our 3D triculture model further supports the importance of including supportive cells to facilitate in vitro functionality and maturation. The incorporation of human iPSC-HPCs in our model also demonstrates the potential for our platform to be used for personalized medicine as a patientspecific approach to study various pathophysiological disease mechanisms, drug screening and discovery applications, as well as for clinical translation. 


\section{3D BIOPRINTING OF DECELLULARIZED EXTRACELLULAR MATRIX AS NEXT- GENERATION BIOINKS}

With the growing advancements in 3D bioprinting technology, the generation of novel biomaterials to serve as cell supportive bioinks is critical to better mimic the native niche microenvironment. In vivo, cells actively interact with the surrounding extracellular matrix (ECM), which functions both as a structural support and a rich reservoir for biochemical constituents including growth factors, cell-binding proteins, collagens, and proteoglycans ${ }^{25}$. In turn, these cell-ECM interactions regulate cell behavior such as proliferation, differentiation, maturation, and migration ${ }^{25}$. Studies characterizing the ECM have also elucidated its diversity such that they vary compositionally between different tissues and organs ${ }^{26,27}$. As such, the hypothesis that recapitulating the tissue-specific ECM will improve cell response and behavior has served as a foundational basis for the development of novel biomaterials.

To address the challenge of creating a tissue-specific ECM, an attractive approach is to preserve the inherent complex native ECM constituents using a decellularization process. In general, tissues and organs are decellularized through a combination of detergent and enzymatic washes as well as mechanical disruption to yield a ECM scaffold that is devoid of cellular content. By undergoing this process, many protocols to date have been established to form a wide range of decellularized tissues including the heart, liver, kidney, adipose, bone, and cartilage ${ }^{28-33}$. Importantly, in vitro studies have also demonstrated that tissue-specific decellularized scaffolds improve the proliferation, differentiation, and maintenance of cell phenotype of cultured tissue-matched cell types ${ }^{26,34,35}$. For instance, ADSCs cultured on 3D decellularized adipose tissue foams showed enhanced adipogenic differentiation in terms of lipid accumulation, GPDH activity, and upregulation of the key adipogenic genes $P P A R \gamma, C E B P \alpha$, and $L P L$ compared to $2 \mathrm{D}$ controls ${ }^{36}$. This is also further supported by the increased proliferation and maintenance of stem cell phenotype of ADSCs expanded on decellularized adipose tissue microcarriers over long term culture compared to collagen-based Cultispher-S ${ }^{\circledR}$ microcarrier controls ${ }^{34}$. While the exact mechanism is unknown, it is believed that the complex nature of the tissue-specific ECM provides an environment more similar to their natural in vivo microenvironment and therefore contributes as an improved cell-instructive substrate. This observation is further supported by a study demonstrating enhanced cell adhesion and proliferation as well as phenotype maintenance when liver, muscle, and skin cells were grown on tissue-matched decellularized ECM coatings versus non-tissue matched controls ${ }^{26}$.

As such, the application of decellularized tissues to serve as tissue-specific bioinks in 3D bioprinting would have great potential towards the advancements of producing biomimetic tissues and organs. Currently, the selection of naturallyderived bioinks available for 3D bioprinting has been limited to biomaterials such as gelatin, collagen, alginate, fibrin, chitosan, and hyaluronic acid ${ }^{37}$, which are simple in composition compared to the native ECM. By developing printable decellularized tissue bioinks compatible with different 3D bioprinters (e.g. extrusion-based or DLP-based printers), the generation of a large library of bioinks tailored for various engineered tissues and organs can be readily accomplished ${ }^{38}$. In particular, it can be envisioned that these tissue-specific decellularized ECM bioinks could be used directly in their pure form or incorporated with other existing bioinks to enhance their functional properties. Overall, decellularized ECM offers a unique strategy to be able to preserve the innate complexity of the tissue-specific microenvironment with potential to improve the functionality of biomimetic tissues that would otherwise be difficult to achieve using a bottom-up approach.

\section{CONCLUSIONS}

In summary, 3D bioprinting technology in recent years have led to the breakthrough development of multiple tissue models for a wide range of applications in tissue engineering and regenerative medicine. More specifically, using our DLP-based 3D bioprinters we developed novel approaches to rapidly fabricate complex tissue structures that possess microscale resolution and physiologically relevant architectures. Namely, we were able to directly print cellularized hierarchal branched vascular networks that were able to support host anastomosis and blood perfusion in vivo, which demonstrates the potential clinical translation of bioprinted tissues. Our lab has also developed the first human biomimetic 3D hepatic triculture model composed of human iPSC-derived hepatic progenitor cells for use as a clinically-relevant platform for personalized medicine and drug screening applications. In general, the future direction of 3D bioprinting functional tissues and organs will involve the incorporation of multiple facets namely the inclusion of heterogeneous cell populations placed in their anatomical locale, intricate vascular networks to maintain long term growth and maturation of the tissue, and the incorporation of bioactive naturally-derived bioinks, such as decellularized ECM, to provide a complex tissue-specific microenvironment to enhance functionality. 


\section{ACKNOWLEDGEMENTS}

We thank the grant support from the National Institutes of Health (R33HD090662 and R01EB021857), and National Science Foundation (1903933 and1644967) to S.C.

\section{REFERENCES}

[1] Ma, X., Qu, X., Zhu, W., Li, Y.-S., Yuan, S., Zhang, H., Liu, J., Wang, P., Lai, C. S. E., Zanella, F., Feng, G.-S., Sheikh, F., Chien, S. and Chen, S., "Deterministically patterned biomimetic human iPSC-derived hepatic model via rapid 3D bioprinting," Proceedings of the National Academy of Sciences 113(8), 2206-2211 (2016).

[2] Lind, J. U., Busbee, T. A., Valentine, A. D., Pasqualini, F. S., Yuan, H., Yadid, M., Park, S.-J., Kotikian, A., Nesmith, A. P., Campbell, P. H., Vlassak, J. J., Lewis, J. A. and Parker, K. K., "Instrumented cardiac microphysiological devices via multimaterial three-dimensional printing," Nature Materials 16(3), 303-308 (2016).

[3] Eisenstein, M., “Artificial organs: Honey, I shrunk the lungs,” Nature 519(7544), S16-S18 (2015).

[4] Homan, K. A., Kolesky, D. B., Skylar-Scott, M. A., Herrmann, J., Obuobi, H., Moisan, A. and Lewis, J. A., "Bioprinting of 3D Convoluted Renal Proximal Tubules on Perfusable Chips," Scientific Reports 6(1), 34845 (2016).

[5] Zhu, W., Qu, X., Zhu, J., Ma, X., Patel, S., Liu, J., Wang, P., Sun, C., Lai, E., Gou, M., Xu, Y., Zhang, K. and Chen, S., "Direct 3D bioprinting of prevascularized tissue constructs with complex microarchitecture," Biomaterials 124, 106-115 (2017).

[6] Nguyen, D., Hägg, D. A., Forsman, A., Ekholm, J., Nimkingratana, P., Brantsing, C., Kalogeropoulos, T., Zaunz, S., Concaro, S., Brittberg, M., Lindahl, A., Gatenholm, P., Enejder, A. and Simonsson, S., "Cartilage Tissue Engineering by the 3D Bioprinting of iPS Cells in a Nanocellulose/Alginate Bioink," Scientific Reports 7(1), 658 (2017).

[7] Kuo, C.-Y., Eranki, A., Placone, J. K., Rhodes, K. R., Aranda-Espinoza, H., Fernandes, R., Fisher, J. P. and Kim, P. C. W., "Development of a 3D Printed, Bioengineered Placenta Model to Evaluate the Role of Trophoblast Migration in Preeclampsia," ACS Biomaterials Science \& Engineering 2(10), 1817-1826 (2016).

[8] Wilson, W. C. and Boland, T., "Cell and organ printing 1: Protein and cell printers," The Anatomical Record 272A(2), 491-496 (2003).

[9] Xu, T., Zhao, W., Zhu, J.-M., Albanna, M. Z., Yoo, J. J. and Atala, A., "Complex heterogeneous tissue constructs containing multiple cell types prepared by inkjet printing technology," Biomaterials 34(1), 130-139 (2013).

[10] Blaeser, A., Duarte Campos, D. F., Puster, U., Richtering, W., Stevens, M. M. and Fischer, H., "Controlling Shear Stress in 3D Bioprinting is a Key Factor to Balance Printing Resolution and Stem Cell Integrity," Advanced Healthcare Materials 5(3), 326-333 (2016).

[11] Murphy, S. V and Atala, A., "3D bioprinting of tissues and organs," Nature Biotechnology 32(8), 773-785 (2014).

[12] Zhang, W., Han, L.-H. and Chen, S., "Integrated Two-Photon Polymerization With Nanoimprinting for Direct Digital Nanomanufacturing," Journal of Manufacturing Science and Engineering 132(3), 030907 (2010).

[13] Paz, V. F., Emons, M., Obata, K., Ovsianikov, A., Peterhänsel, S., Frenner, K., Reinhardt, C., Chichkov, B., Morgner, U. and Osten, W., "Development of functional sub-100 nm structures with 3D two-photon polymerization technique and optical methods for characterization," Journal of Laser Applications 24(4), 042004 (2012).

[14] Zhang, A. P., Qu, X., Soman, P., Hribar, K. C., Lee, J. W., Chen, S. and He, S., "Rapid Fabrication of Complex 3D Extracellular Microenvironments by Dynamic Optical Projection Stereolithography," Advanced Materials 24(31), 4266-4270 (2012).

[15] Tumbleston, J. R., Shirvanyants, D., Ermoshkin, N., Janusziewicz, R., Johnson, A. R., Kelly, D., Chen, K., Pinschmidt, R., Rolland, J. P., Ermoshkin, A., Samulski, E. T. and DeSimone, J. M., “Continuous liquid interface production of 3D objects," Science 347(6228), 1349-1352 (2015).

[16] Lovett, M., Lee, K., Edwards, A. and Kaplan, D. L., "Vascularization strategies for tissue engineering.," Tissue engineering. Part B, Reviews 15(3), 353-370 (2009). 
[17] Fischbach, C. and Mooney, D. J., "Polymers for pro- and anti-angiogenic therapy.," Biomaterials 28(12), 20692076 (2007).

[18] Lee, K. Y., Peters, M. C., Anderson, K. W. and Mooney, D. J., "Controlled growth factor release from synthetic extracellular matrices," Nature 408(6815), 998-1000 (2000).

[19] Chen, X., Aledia, A. S., Ghajar, C. M., Griffith, C. K., Putnam, A. J., Hughes, C. C. W. and George, S. C., "Prevascularization of a Fibrin-Based Tissue Construct Accelerates the Formation of Functional Anastomosis with Host Vasculature," Tissue Engineering Part A 15(6), 1363-1371 (2009).

[20] Thorne, J. T., Segal, T. R., Chang, S., Jorge, S., Segars, J. H. and Leppert, P. C., "Dynamic Reciprocity Between Cells and Their Microenvironment in Reproduction1," Biology of Reproduction 92(1) (2015).

[21] Kaur, G. and Dufour, J. M., "Cell lines: Valuable tools or useless artifacts.," Spermatogenesis 2(1), 1-5 (2012).

[22] Martins-Taylor, K. and Xu, R.-H., "Concise Review: Genomic Stability of Human Induced Pluripotent Stem Cells," STEM CELLS 30(1), 22-27 (2012).

[23] Guengerich, F. P., "Cytochrome P-450 3A4: Regulation and Role in Drug Metabolism," Annual Review of Pharmacology and Toxicology 39(1), 1-17 (1999).

[24] Faucette, S. R., Wang, H., Hamilton, G. A., Jolley, S. L., Gilbert, D., Lindley, C., Yan, B., Negishi, M. and LeCluyse, E. L., "Regulation of CYP2B6 in primary human hepatocytes by prototypical inducers," Drug Metabolism and Disposition 32(3), 348-358 (2004).

[25] Crapo, P. M., Gilbert, T. W. and Badylak, S. F., "An overview of tissue and whole organ decellularization processes," Biomaterials 32(12), 3233-3243 (2011).

[26] Zhang, Y., He, Y., Bharadwaj, S., Hammam, N., Carnagey, K., Myers, R., Atala, A. and Van Dyke, M., "Tissuespecific extracellular matrix coatings for the promotion of cell proliferation and maintenance of cell phenotype," Biomaterials 30(23-24), 4021-4028 (2009).

[27] Russo, V., Yu, C., Belliveau, P., Hamilton, A. and Flynn, L. E., "Comparison of Human Adipose-Derived Stem Cells Isolated from Subcutaneous, Omental, and Intrathoracic Adipose Tissue Depots for Regenerative Applications," STEM CELLS Translational Medicine 3(2), 206-217 (2014).

[28] Ott, H. C., Matthiesen, T. S., Goh, S.-K., Black, L. D., Kren, S. M., Netoff, T. I. and Taylor, D. A., "Perfusiondecellularized matrix: using nature's platform to engineer a bioartificial heart," Nature Medicine 14(2), 213-221 (2008).

[29] Mazza, G., Rombouts, K., Rennie Hall, A., Urbani, L., Vinh Luong, T., Al-Akkad, W., Longato, L., Brown, D., Maghsoudlou, P., Dhillon, A. P., Fuller, B., Davidson, B., Moore, K., Dhar, D., De Coppi, P., Malago, M. and Pinzani, M., "Decellularized human liver as a natural 3D-scaffold for liver bioengineering and transplantation," Scientific Reports 5(1), 13079 (2015).

[30] Yu, Y. L., Shao, Y. K., Ding, Y. Q., Lin, K. Z., Chen, B., Zhang, H. Z., Zhao, L. N., Wang, Z. B., Zhang, J. S., Tang, M. L. and Mei, J., "Decellularized kidney scaffold-mediated renal regeneration," Biomaterials 35(25), 6822-6828 (2014).

[31] Flynn, L. E., "The use of decellularized adipose tissue to provide an inductive microenvironment for the adipogenic differentiation of human adipose-derived stem cells," Biomaterials 31(17), 4715-4724 (2010).

[32] Chen, G. and Lv, Y., "Decellularized Bone Matrix Scaffold for Bone Regeneration," [Methods in molecular biology (Clifton, N.J.)] (2017).

[33] Schwarz, S., Koerber, L., Elsaesser, A. F., Goldberg-Bockhorn, E., Seitz, A. M., Dürselen, L., Ignatius, A., Walther, P., Breiter, R. and Rotter, N., "Decellularized cartilage matrix as a novel biomatrix for cartilage tissueengineering applications.," Tissue engineering. Part A 18(21-22), 2195-2209 (2012).

[34] Yu, C., Kornmuller, A., Brown, C., Hoare, T. and Flynn, L. E., "Decellularized adipose tissue microcarriers as a dynamic culture platform for human adipose-derived stem/stromal cell expansion," Biomaterials 120, 66-80 (2017).

[35] Fong, A. H., Romero-López, M., Heylman, C. M., Keating, M., Tran, D., Sobrino, A., Tran, A. Q., Pham, H. H., Fimbres, C., Gershon, P. D., Botvinick, E. L., George, S. C. and Hughes, C. C. W., "Three-Dimensional Adult Cardiac Extracellular Matrix Promotes Maturation of Human Induced Pluripotent Stem Cell-Derived Cardiomyocytes.," Tissue engineering. Part A 22(15-16), 1016-1025 (2016).

[36] Yu, C., Bianco, J., Brown, C., Fuetterer, L., Watkins, J. F., Samani, A. and Flynn, L. E., "Porous decellularized adipose tissue foams for soft tissue regeneration,” Biomaterials 34(13), 3290-3302 (2013).

[37] Chia, H. N. and Wu, B. M., "Recent advances in 3D printing of biomaterials," Journal of Biological Engineering 9(1), 4 (2015). 
[38] Pati, F., Jang, J., Ha, D.-H., Won Kim, S., Rhie, J.-W., Shim, J.-H., Kim, D.-H. and Cho, D.-W., "Printing threedimensional tissue analogues with decellularized extracellular matrix bioink," Nature Communications 5, 3935 (2014).

Proc. of SPIE Vol. 11294 1129405-12

Downloaded From: https://www.spiedigitallibrary.org/conference-proceedings-of-spie on 25 Apr 2023 Terms of Use: https://www.spiedigitallibrary.org/terms-of-use 